\title{
How Many Squares Must a Binary Sequence Contain?
}

\author{
Aviezri S. Fraenkel ${ }^{1}$ and R. Jamie Simpson ${ }^{2}$ \\ Submitted: November 16, 1994; Accepted: December 11, 1994
}

AbStract. Let $g(n)$ be the length of a longest binary string containing at most $n$ distinct squares (two identical adjacent substrings). Then $g(0)=3(010$ is such a string), $g(1)=7$ (0001000) and $g(2)=18$ (010011000111001101). How does the sequence $\{g(n)\}$ behave? We give a complete answer.

\section{Introduction}

A binary word (or string) containing no square (a pair of identical adjacent subwords) has maximum length 3 ; in fact, the only squarefree words of length 3 are 010 and its 1-complement 101. A computer disclosed that a binary word containing at most 1 square has maximum length 7: the only words of length 7 with only 1 square are

$$
\text { 0001000, } \quad 0100010, \quad 0111011
$$

and their 1-complements and the reverse of 0111011 and its 1-complement. Further, a binary word containing at most 2 distinct squares has maximum length 18; the only words of length 18 which contain only 2 distinct squares are

$$
010011000111001101
$$

and its 1-complement (which is also its reverse).

In general, let $g(k)$ denote the length of a longest binary word containing at most $k$ distinct squares. "Distinct" means that the squares are of different shape, not just translates of each other. We have seen that $g(0)=3, g(1)=7, g(2)=18$. This data raises the following natural questions.

1 Department of Applied Mathematics \& Computer Science, The Weizmann Institute of Science, Rehovot 76100, Israel. Email: fraenkel@wisdom.weizmann.ac.il . Work done while visiting Curtin University.

2 School of Mathematics, Curtin University, Perth WA 6001, Australia. Email: tsimpsonr@cc.curtin.edu.au 
1. Is the set of values of the sequence $S=\{g(k): k=0,1, \ldots\}$ infinite or finite?

2. What's the value of $g(3)$ ?

Regarding the first of these questions, Entringer, Jackson and Schatz [1974] considered the conjecture that $S$ is infinite, citing a reference "which... seems to say that [this] conjecture... is true". They then go on to show that $S$ is finite, by proving that $g(5)=\infty$, i.e., there exists an infinite binary sequence with only 5 squares!

It has been shown many times that there exist infinite squarefree ternary sequences. See e.g., Thue [1912], Morse and Hedlund [1944], Hawkins and Mientka [1956], Leech [1957], Novikov and Adjan [1968], Pleasants [1970], Burris and Nelson [1971/72], del Junco [1977], Ehrenfeucht and Rozenberg [1983]. (Currie [1993] wrote: "One reason for this sequence of rediscoveries is that nonrepetitive sequences have been used to construct counterexamples in many areas of mathematics: ergodic theory, formal language theory, universal algebra and group theory, for example...".) Actually, Thue [1912] showed more: there exists a doubly infinite squarefree ternary sequence which also avoids the 2 triples $a_{1} a_{3} a_{1}$ and $a_{2} a_{3} a_{2}$. See Berstel $[1992, \S 4.2]$ for an exposition of the full result, and Berstel [ $\geq 1995]$ for an English translation of Thue's papers.

Roth [1991] has proved that given any alphabet $\Sigma$ of more than 2 letters, any given pattern, such as a square, is avoidable over $\Sigma$, if and only if there exists an infinite binary word in which any morphism of that pattern is of bounded length.

Seen in this light, the result of Entringer et al. [1974] is not surprising. But it brings into even sharper focus the second question, because it makes us wonder about the values of $g(3)$ and $g(4)$.

We give a complete answer by showing that $g(3)=\infty$. In $\S 2$, after establishing some notation and definitions, we construct an infinite binary sequence, and in $\S 3$ we prove that it contains only the 3 squares $0^{2}, 1^{2}$ and $(01)^{2}$.

We also remark that questions regarding squares in sequences arise in molecular biology, where they are known as repeats, or tandem repeats. In fact, the most frequent repeat in the human genome seems to be the binary word GT, with high copy number (the number of times GT is repeated). Trifonov [1989] argues that the copy number influences the functions of DNA chains adjacent to the repeated word, such as their binding power and gene expression; it can even cause certain diseases if too high or too low; and it also influences the unwinding capability of the DNA helix. Algorithms for identifying repeats and databases of repeats in the human genome are maintained by Milosavljević $[\geq 1995]$.

Since the copy number at a given site changes from one individual to another, the copy number has also been used in DNA-fingerprinting. This application appears to have been originated by Alec Jeffreys' group in Leicester. See e.g., Jeffreys, 
Wilson and Thein [1985] and Jeffreys, Turner and Debenham [1991]. Further elaborations on applications of DNA-fingerprinting to medicine and forensic medicine are given in Raskó and Downes [1995, ch. 6, especially p. 156; and ch. 12, especially pp. 379-380], where it is also stated that the human genome contains some 500,000 repeated words. (Keywords for human genome applications are VNTR (Variable Number Tandem Repeats) and mini- and microsatellite sequences for the basic subwords that are repeated.)

\section{Construction of the Binary Sequence}

We begin with some notation.

Denote by $\Sigma^{*}$ the set of all words (finite or infinite strings, also called blocks) over the finite alphabet $\Sigma$, whose elements are letters. Given a finite word $\sigma=$ $\sigma_{1} \cdots \sigma_{n} \in \Sigma^{*}, \sigma_{i} \in \Sigma(i \in\{1, \ldots, n\})$, the length of $\sigma$ is $|\sigma|=n=$ number of letters in $\sigma$, counting multiplicities. Below we use the binary, ternary and quinary alphabets, denoted by $B=\{0,1\}, T=\left\{a_{1}, a_{2}, a_{3}\right\}, Q=\left\{a_{1}, a_{2}, a_{3}, a_{4}, a_{5}\right\}$, respectively.

A prefix of a word is a subword at the beginning (left side) of the word; a suffix is a subword at the end (right side) of the word. Given words $x, y \in \Sigma^{*}$, we denote by $x y$ the concatenation of these words, beginning with $x$ and ending with $y$. Thus $x^{2}$ is the square $x x$. If $x$ is a subword of $y$, we also write $x \subseteq y$.

A function $C: Q^{*} \rightarrow B^{*}$ is an encoding (a binary encoding of $Q^{*}$ ). Given a finite or infinite quinary word $q=q_{1} q_{2} \cdots \in Q^{*}, q_{i} \in Q(i \in\{1,2, \ldots\}), C$ is defined by the code $C(q)=C\left(q_{1}\right) C\left(q_{2}\right) \cdots$, where the $C\left(a_{i}\right)$ are the given codewords $(i \in\{1, \ldots, 5\})$. Thus the codeword $C\left(a_{i}\right)$ is also the code of $a_{i}$. Decoding refers to the inverse function $C^{-1}: B^{*} \rightarrow Q^{*}$ if it exists. To parse any subword of a code means to identify beginnings and ends of all the codewords contained entirely in the subword.

We are now ready to describe the construction of the doubly infinite binary word which has only 3 squares. Since the construction involves infinite processes, we call it a procedure rather than an algorithm.

Procedure TQB. (1) Let $t \in T^{*}$ be a doubly infinite squarefree ternary word over $T=\left\{a_{1}, a_{2}, a_{3}\right\}$, which avoids $a_{1} a_{3} a_{1}$ and $a_{2} a_{3} a_{2}$.

(2) Replace every occurrence of $a_{2} a_{3}$ in $t$ by $a_{2} a_{4} a_{3}$, and every occurrence of $a_{3} a_{2}$ by $a_{3} a_{5} a_{2}$. The result is a doubly infinite quinary word $q \in Q^{*}$. 
TABle 1. Possible pairs of $q$.

$a_{1} a_{2} \quad a_{2} a_{1} \quad a_{3} a_{1} \quad a_{4} a_{3}$

$a_{1} a_{3} \quad a_{2} a_{4} \quad a_{3} a_{5} \quad a_{5} a_{2}$

TABle 2. Possible triples of $q$.

$\begin{array}{llll}a_{1} a_{2} a_{1} & a_{2} a_{1} a_{2} & a_{3} a_{1} a_{2} & a_{4} a_{3} a_{1} \\ a_{1} a_{2} a_{4} & a_{2} a_{1} a_{3} & a_{3} a_{1} a_{3} & a_{5} a_{2} a_{1} \\ a_{1} a_{3} a_{5} & a_{2} a_{4} a_{3} & a_{3} a_{5} a_{2} & a_{5} a_{2} a_{4}\end{array}$

(3) Define $C(q)$ by

$$
\begin{aligned}
& C\left(a_{1}\right)=011000111001 \\
& C\left(a_{2}\right)=011100011001 \\
& C\left(a_{3}\right)=011001110001 \\
& C\left(a_{4}\right)=01100010111001 \\
& C\left(a_{5}\right)=01110010110001 .
\end{aligned}
$$

From this encoding we see that $C(q)$ contains the squares $0^{2}, 1^{2}$ and $(01)^{2}$. In the next section we show that $C(q)$ contains no other squares. The main idea is to establish an explicit bound on the length of the squares of $C(q)$. \{ The name TQB of the procedure of course reminds us that in step 1 we have a Ternary sequence, in step 2 we create a Quinary sequence, and in step 3 a Binary sequence.

\section{The Binary Sequence Contains Only 3 Squares}

A single 0 sandwiched between 2 neighboring 1-bits will be called an isolated 0 .

We begin by collecting some easily proved properties of the sequences $q$ and $C(q)$ generated in Procedure TQB.

(i) All and only all the pairs and triples of $q$ are listed in Tables 1 and 2 respectively.

(ii) The lengths of the $C\left(a_{i}\right)$ is $12(i \in\{1,2,3\})$ and $14(i \in\{4,5\})$. Only $C\left(a_{4}\right)$ and $C\left(a_{5}\right)$ contain isolated 0's; the only other isolated 0's are at the beginning of every codeword $C\left(a_{i}\right)$, in every concatenation $C\left(a_{j}\right) C\left(a_{i}\right)$. Hence the only distances between consecutive isolated 0's in $C(q)$ are 7 or 12 . The sequence of these distances has the form

$$
\begin{gathered}
\cdots 7^{2} 12^{r_{-2}} 7^{2} 12^{r_{-1}} 7^{2} 12^{r_{0}} 7^{2} 12^{r_{1}} 7^{2} 12^{r_{2}} 7^{2} \cdots, \\
-4- \\
4^{-}
\end{gathered}
$$


where the $r_{i}$ are positive integers (since $a_{4}$ and $a_{5}$ cannot be adjacent).

(iii) The doubly infinite sequence $C(q)$ can be parsed uniquely into codewords $C\left(a_{i}\right)(i \in\{1, \ldots, 5\})$ by placing a comma in front of isolated 0's at distances 12 and 14 (skipping those isolated 0's which are at distance 7 from both of their preceding and succeeding isolated 0 ). Thus $C(q)$ can be decoded uniquely into $q$.

(iv) A codeword $C\left(a_{i}\right)$ is not a prefix or suffix of $C\left(a_{j}\right)$ for any $j \neq i$.

We show now that property (iii) can be strengthened: also certain finite, even short subwords of $C(q)$ can be parsed uniquely.

Proposition 1. Any subword $w$ of $C(q)$ which contains a codeword can be parsed uniquely, and so any codeword in $w$ can be decoded uniquely.

Proof. Suppose first that $w$ contains no isolated 0. Then (ii) implies that $|w|=12$ or 13 , and the 12 left bits constitute a unique codeword. If $w$ contains 2 isolated 0's at distance 12 then a unique codeword at length 12 can be identified, which induces a unique parsing on $w$. Unique parsing also results if $w$ contains 3 isolated 0's at distances 7, 7, when a unique codeword of length 14 can be identified. By (ii), the only remaining cases are 2 isolated 0 's, $z_{1}$ and $z_{2}$, at distance 7 , say with $z_{1}$ to the left of $z_{2}$, or else a single isolated 0 , denoted by $z$.

If there are precisely 12 bits to the left of $z_{1}$ (or $z$ ), then they constitute a unique codeword. Similarly, if there are 11 or 12 bits to the right of $z_{2}$ (or $z$ ), then $z_{2}$ (or $z$ ) and the first 11 bits to its right constitute a unique codeword. So suppose that neither of these two cases holds. Then $w$ must contain $C\left(a_{4}\right)$ or $C\left(a_{5}\right)$. In fact, either there are precisely 7 bits to the left of $z_{1}$ beginning in 01 , which constitute the beginning of $C\left(a_{4}\right)$ or $C\left(a_{5}\right)$; or else there are precisely 6 or 7 bits to the right of $z_{2}$, the first 6 of which end in 01 , which constitute the end of $C\left(a_{4}\right)$ or $C\left(a_{5}\right)$. In the case of $z$, there must be precisely 7 bits to the left of $z$ beginning in 011 and precisely 6 or 7 bits to the right of $z$, the first 6 of which end in 001, which identifies $C\left(a_{4}\right)$ or $C\left(a_{5}\right)$ uniquely.

In Table 3 the braces indicate illegal parsings; in fact, they violate the conditions, given at the end of the proof, which the bits near $z_{1}, z_{2}$ and $z$ have to satisfy. By (i), Table 3 lists all the pairs containing $a_{4}$ or $a_{5}$.

We now come to the main result.

Proposition 2. Let $C(q)$ be a doubly infinite binary word produced by Procedure TQB. Then every square of $C(q)$ is contained in some subword $C\left(q^{\prime}\right) \subseteq$ $C(q)$ where $q^{\prime} \subseteq q$ with $\left|q^{\prime}\right| \leq 3$.

ProOF. Suppose $b_{1} \cdots b_{2 m} \subseteq C\left(q^{\prime}\right)$ is a (binary) square which intersects the code of $\left|q^{\prime}\right| \geq 4$ letters of $q$. Denote the words $b_{1} \cdots b_{m}, b_{m+1} \cdots b_{2 m}, b_{1} \cdots b_{2 m}$ by $w_{L}, w_{R}, w=w_{L} w_{R}$ respectively. Observe that $\left|q^{\prime}\right| \geq 4$ implies that either $w_{L}$ or $w_{R}$ contains a complete codeword, say $c_{1}$. Assume $c_{1}$ is contained in $w_{L}$, say. 
TABLE 3. Encodings of the 4 pairs containing $a_{4}$ and $a_{5}$.

$$
\begin{aligned}
& C\left(a_{2} a_{4}\right)=01110 \overbrace{011001 \mid 0110001} 0111001 \\
& C\left(a_{3} a_{5}\right)=01100 \overbrace{111001 \mid 0111001} 0110001 \\
& C\left(a_{4} a_{3}\right)=0110001 \overbrace{0111001 \mid 0110011}^{0} 001 \\
& C\left(a_{5} a_{2}\right)=0111001 \overbrace{0110001 \mid 0111000}^{0} 0011
\end{aligned}
$$

Suppose first that the leftmost bit of $c_{1}$ is at $b_{1}$. Since $w$ is a square, the bits of $c_{1}$ appear also in $w_{R}$, with the leftmost bit at $b_{m+1}$. By (iv) and Proposition 1 , actually $c_{1}$ appears in $w_{R}$, left-justified, and the complement of of this left-justified $c_{1}$ with respect to $w_{R}$ is tiled uniquely with an integer number of codewords $c_{i}$. The same codewords then appear, shifted left by $m$ places, in the complement of the left-justified $c_{1}$ of $w_{L}$ with respect to $w_{L}$. Since the parsing is unique and $w$ contains no part-codewords, the decoding exists, and so $q$ contained a square, which is a contradiction. The same contradiction results if we assume that the rightmost bit of $c_{1}$ is at position $b_{m}$.

We may thus assume that $c_{1}$ is neither right- nor left-justified in $w_{L}$. Without loss of generality we may assume that $c_{1}$ is the leftmost codeword contained entirely in $w_{L}$. Since $w$ is a square, Proposition 1 implies that $c_{1}$ also appears in $w_{R}$, at a unique location, namely right-shifted by $m$ places from its location in $w_{L}$. Thus $c_{1}$ begins at some location $j+1>m+1$, and so at location $j \geq m+1$, a codeword $c_{2}$ ends, which begins at some location $k \leq m$.

Suppose first that at least 8 of the bits of the suffix of $c_{2}$ are in $w_{R}$. We then use the following left-shift argument.

From the mapping $C$ defined in Procedure TQB we see that a suffix of length $\geq 8$ determines $c_{2}$ uniquely, when also the location $j$ of the end of $c_{2}$ is given. (Knowing this location is crucial: note that the suffix of length 13 of $C\left(a_{4}\right)$ is identical to a subword of length 13 contained in the interior of $C\left(a_{3} a_{5}\right)$.) Since $w$ is a square, it follows that at location $j-m \geq 1$ there is the end of the codeword $c_{2}$, which begins at location $k-m<1$.

Again using the fact that $w$ is a square we now have, in particular, $b_{i}=b_{i+m}$ for $i=k-m, \ldots, k-1$, i.e., we have another square

$$
w^{\prime}=b_{k-m} \cdots b_{k-1} b_{k} \cdots b_{k+m-1}=w_{L}^{\prime} w_{R}^{\prime}
$$

also of length $2 m$, shifted left of $w$ by $m-k$ bits, where $w_{L}^{\prime}=b_{k-m} \cdots b_{k-1}$ and $w_{R}^{\prime}=b_{k} \cdots b_{k+m-1}$. Now $w_{R}^{\prime}$ begins with a codeword and ends with one. As we 
saw above this implies that $q$ has a square, which is a contradiction. This ends the left-shift argument.

We end the proof by considering four cases for the length of the suffix of $c_{2}$.

I. Assume that $c_{2}$ has a suffix of precisely 7 bits in $w_{R}$. The mapping $C$ reveals that then $c_{2}$ is uniquely determined, except when $c_{2}=C\left(a_{1}\right)$ or $C\left(a_{4}\right)$. When $c_{2}$ is uniquely determined, then the left-shift argument applies as above.

So assume first that $c_{2}=C\left(a_{1}\right)$. If $C\left(a_{1}\right)$ intersects also the beginning of $w_{L}$, then the left-shift argument applies. Thus assume $C\left(a_{4}\right)$ intersects the beginning of $w_{L}$. By Table 1, $C\left(a_{4}\right)$ is followed by $C\left(a_{3}\right)$. Since $w$ is a square, $C\left(a_{3}\right)$ must follow $C\left(a_{1}\right)$ in $w_{R}$. By Table 2 , this $C\left(a_{3}\right)$ must be followed by $C\left(a_{5}\right)$. If this $C\left(a_{5}\right)$ is contained in $w_{R}$, then $C\left(a_{5}\right)$ must follow $C\left(a_{3}\right)$ in $w_{L}$. Thus $C\left(a_{4} a_{3} a_{5}\right)$ intersects $w_{L}$. This is a contradiction, since the triple $a_{4} a_{3} a_{5}$ doesn't appear in Table 2 (since $t$ doesn't contain $a_{2} a_{3} a_{2}$ ). If $C\left(a_{5}\right)$ is not contained entirely in $w_{R}$, then the end of $C\left(a_{3}\right)$ and the beginning of $C\left(a_{1}\right)$ in $w_{L}$ are adjacent bits. Since $w$ is a square, the first 5 bits of $C\left(a_{1}\right)$ and $C\left(a_{5}\right)$ must then agree, but they don't.

Secondly, assume that $c_{2}=C\left(a_{4}\right)$. If $C\left(a_{4}\right)$ also intersects the beginning of $w_{L}$, the left-shift argument applies. So assume that $C\left(a_{1}\right)$ intersects the beginning of $w_{L}$. By Table 2, $C\left(a_{4}\right)$ is followed by $C\left(a_{3} a_{1}\right)$ (since $a_{3} a_{2}$ cannot appear in $q)$. Note that $C\left(a_{3}\right)$ must then be contained in both $w_{R}$ and $w_{L}$. If $C\left(a_{3} a_{1}\right)$ is contained in $w_{R}$, then $C\left(a_{3} a_{1}\right)$ also appears after $C\left(a_{1}\right)$ in $w_{L}$. But then $q$ and hence $t$ contained $a_{1} a_{3} a_{1}$, which is a contradiction. If $C\left(a_{1}\right)$ is not contained entirely in $w_{R}$, then the end of $C\left(a_{3}\right)$ and the beginning of $C\left(a_{4}\right)$ in $w_{L}$ must be adjacent bits. This is impossible, since $q$ doesn't contain $a_{3} a_{4}$.

II. Assume that $c_{2}$ has a suffix of precisely 6 bits in $w_{R}$. Then case I applies a fortiori, and the same proof is valid. But now, in addition, $C\left(a_{3}\right)$ and $C\left(a_{5}\right)$ have the same suffix (of 6 bits).

Assume first that $c_{2}=C\left(a_{3}\right)$. The only case that needs to be considered is when $C\left(a_{5}\right)$ intersects the beginning of $w_{L}$. It is followed by $C\left(a_{2}\right)$ (Table 1$)$. Then $C\left(a_{2}\right)$ follows $C\left(a_{3}\right)$ in $w_{R}$, which is a contradiction, since $q$ doesn't contain $a_{3} a_{2}$.

Secondly, assume that $c_{2}=C\left(a_{5}\right)$. Then $C\left(a_{5}\right)$ has a prefix of length 8 in $w_{L}$, which is seen to be unique, so a right-shift argument, analogous to the left-shift argument, applies.

III. Assume that $c_{2}$ has a suffix of precisely 5 bits in $w_{R}$. Then case II applies a fortiori, but also $C\left(a_{1}\right), C\left(a_{2}\right)$ and $C\left(a_{4}\right)$ have the same suffix (of 5 bits).

Suppose first that $c_{2}=C\left(a_{1}\right)$ and $C\left(a_{2}\right)$ intersects the beginning of $w_{L}$. Now Table 1 shows that $C\left(a_{2}\right)$ is followed by $C\left(a_{1}\right)$ or $C\left(a_{4}\right)$. The former is impossible since then $q$ contains the square $a_{1}^{2}$, and the latter is impossible since then $q$ contains $a_{1} a_{4}$. So assume $c_{2}=C\left(a_{2}\right)$ and $C\left(a_{1}\right)$ intersects the beginning of $w_{L}$. 
Now $C\left(a_{1}\right)$ is followed either by $C\left(a_{2}\right)$ or $C\left(a_{3}\right)$. The former is impossible, since $q$ doesn't contain a square $a_{2}^{2}$, and the latter is impossible since $q$ doesn't contain $a_{2} a_{3}$.

Secondly, assume that $c_{2}=C\left(a_{2}\right)$ and $C\left(a_{4}\right)$ intersects the beginning of $w_{L}$. Now $C\left(a_{4}\right)$ is followed by $C\left(a_{3}\right)$, so $C\left(a_{3}\right)$ must follow $C\left(a_{2}\right)$ in $w_{R}$, which is impossible, since $q$ doesn't contain $a_{2} a_{3}$. If $c_{2}=C\left(a_{4}\right)$ and $C\left(a_{2}\right)$ intersects the beginning of $w_{L}$, we get the same contradiction.

IV. Assume that $c_{2}$ has a suffix of $\leq 4$ bits in $w_{R}$. Then $c_{2}$ has a prefix of $\geq 8$ bits at the end of $w_{L}$ which determines $c_{2}$ uniquely, so a right-shift argument applies.

Thus the assumption $\left|q^{\prime}\right| \geq 4$ leads to a contradiction in all cases, hence $\left|q^{\prime}\right| \leq 3$.

A computer program verified that for all the triples in Table 3, the only squares in the code of these triples are the obvious ones: $0^{2}, 1^{2}$ and $(01)^{2}$. This completes our proof that $g(3)=\infty$.

Acknowledgment. We would like to thank Justin Carpenter for his invaluable help with the computations.

\section{REFERENCES}

1. J. Berstel [1992], Axel Thue's work on repetitions in words, in: Séries Formelles et Combinatoire Algébrique (P. Leroux and C. Reutenauer, eds.), Publ. du LACIM, Vol. 11, Université de Québec, à Montréal, pp. 65-80.

2. J. Berstel $[\geq 1995]$, Axel Thue's papers on repetition in words: an English translation, Publ. du LACIM, Université de Québec, à Montréal.

3. S. Burris and E. Nelson [1971/72], Embedding the dual of $\pi_{\infty}$ in the lattice of equational classes of semigroups, Algebra Universalis 1, 248-153.

4. J.D. Currie [1993], Open problems in pattern avoidance, Amer. Math. Monthly 100, 790-793.

5. A. del Junco [1977], A transformation with simple spectrum which is not rank one, Canad. J. Math. 29, 655-663.

6. A. Ehrenfeucht and G. Rozenberg [1983], On the separating power of EOL systems, RAIRO Inform. Théor. 17, 13-22.

7. R. Entringer, D. Jackson and J. Schatz [1974], On nonrepetitive sequences, J. Combin. Theory (Ser. A) 16, 159-164.

8. D. Hawkins and W.E. Mientka [1956], On sequences which contain no repetitions, Math. Student 24, 185-187. 
9. A.J. Jeffreys, M. Turner and P. Debenham [1991], The efficiency of multilocus DNA fingerprint probes for individualization and establishment of family relationships, determined from extensive casework, Am. J. Hum. Genet. 48, 824-840.

10. A.J. Jeffreys, V. Wilson and S.L. Thein [1985], Hypervariable 'minisatellite' regions in human DNA, Nature 314, 67-73.

11. A.J. Jeffreys, V. Wilson and S.L. Thein [1985], Individual-specific 'fingerprints' of human DNA, Nature 316, 76-79.

12. J.A. Leech [1957], A problem on strings of beads, Math. Gaz. 41, 277-278.

13. A. Milosavljević $[\geq 1995]$, Repeat Analysis, Ch. 13, Sect. 4, Imperial Cancer Research Fund Handbook of Genome Analysis, Blackwell Scientific Publications, in press.

14. M. Morse and G.A. Hedlund [1944], Unending chess, symbolic dynamics and a problem in semigroups, Duke Math. J. 11, 1-7.

15. P.S. Novikov and S.I. Adjan [1968], Infinite periodic groups I, II, III, Izv. Akad. Nauk. SSSR Ser. Mat. 32, 212-244; 251-524; 709-731.

16. P.A.B. Pleasants [1970], Non-repetitive sequences, Proc. Cambridge Phil. Soc. 68, 267-274.

17. I. Raskó and C.S. Downes [1995], Genes in Medicine: Molecular Biology and Human Genetic Disorders, Chapman \& Hall, London.


pos. Theoretical Aspects of Computer Science (STACS; C. Choffrut and M. Jantzen, eds.), Hamburg, Lect. Notes in Comp. Sci. 480, Springer-Verlag, pp. $42-49$.

19. A. Thue [1912], Über die gegenseitige Lage gleicher Teile gewisser Zeichenreihen, Norske Vid. Selsk. Skr., I. Mat. Nat. Kl. Christiania I, 1-67.

20. E.N. Trifonov [1989], The multiple codes of nucleotide sequences, Bull. Math. Biology 51, 417-432. 